\title{
Natural Oscillation Control Experiments on a Prototype Mechanical Rectifier *
}

\author{
Md Nurul Islam* Zhiyong Chen** \\ School of Electrical Engineering and Computer Science \\ University of Newcastle, Callaghan, NSW 2308, Australia \\ *md.n.islam@uon.edu.au \\ ** zhiyong.chen@newcastle.edu.au
}

\begin{abstract}
For a typical biologically inspired multi-segmental mechanical system, the system's locomotion behavior is rectified from the segments' rhythmic oscillation. A central pattern generator $(\mathrm{CPG})$ based controller has been developed to achieve the locomotion with a certain sense of natural oscillation. We designed and manufactured a prototype mechanical rectifier (PMR) system which captures the essential mechanism of multi-segmental systems. Experiments were conducted on the PMR system to reveal the effectiveness of the natural oscillation entrainment controller.
\end{abstract}

Keywords: Oscillation, locomotion, robotics, neuronal control, central pattern generator

\section{INTRODUCTION}

Animal locomotion as the act of self-propulsion by an animal has many manifestations like walking, swimming, crawling, running, jumping and flying. A typical animal locomotion pattern is achieved by rhythmically moving its organisms interacted with environment. Physically, locomotion requires energy to overcome friction, drag, inertia, and gravity, though in many circumstances some of these factors are negligible. The research on exploiting the mechanical resonance between the body and environment for an efficient locomotion can be traced back to Cavagna et al. (1977). The idea of exploiting the natural rhythmic patterns has led to many efficient robotic locomotors that are robust against and adaptive to environmental changes (see, e.g., Hatsopoulos (1996); Williamson (1998); Verdaasdonk et al. (2007); Iwasaki and Zheng (2006); Futakata and Iwasaki (2008); Blair and Iwasaki (2010).) One of the current engineering research focuses on mechanical locomotion behaviors is directed to a class of so-called $m e-$ chanical rectifier systems, which are engineering analogue of various locomotion behaviors of multi-segmental animals including snake, leech, octopus, jellyfish, etc. In this model, it is shown how the change in the body shape (e.g., the segmental link angles) results from the interaction with environment (e.g., the locomotion forward velocity with respect to the inertial frame) and the body actuation. On the contrary, it is also shown how the aforementioned undulatory body shape is rectified to a forward locomotion velocity. These two effects are coupled to form control principles for animal locomotion as designed by nature and for autonomous robotic locomotors in engineering perspective. In this sense, this model is called a mechanical rectifier model. To undercover the fundamental engineering mechanism underlying the rectifier model, the idea of building a prototype mechanical rectifier (PMR) was first proposed

^ The work was supported by the Australian Research Council under Grant No. DP0878724. by Iwasaki and Liu (2004). The PMR model is simple enough to allow us to extract engineering principles by theoretical analysis, simulation, and experiments. At the same time, it captures the essential dynamical mechanism of animal locomotion. The PMR system consists of two main parts: a double pendulum and a disk. The intended operation of the PMR is basically to swing the pendulum to make the disk rotate. The key is that oscillatory motion of the body (pendulum) can generate locomotion (rotation of the disk) if (and only if) the oscillations of body parts are appropriately coordinated. In this paper, we discuss the design and manufacture of a PMR structure and use it as the experimental platform to examine a kind of natural oscillation controller.

For standard mechanical systems described by symmetric positive definite mass, stiffness, and damping matrices, a natural mode of oscillation is defined to be a free response of the modified system obtained by removing all the damping effects to achieve marginal stability for sustained oscillation. However, in typical models of body-environment interactions during locomotion including the PMR model, the environmental forces on the body appear in the equation of motion as terms containing an asymmetric stiffness matrix. In Chen and Iwasaki (2009), the definition of natural oscillation was extended to non-symmetric systems. In particular, it was revealed that a natural oscillation can be achieved by a central pattern generators (CPG) motivated controller which adjusts the damping effect by a proper amount. In fact, biological control mechanisms for animal locomotion are known to consist of CPG (see, e.g., Brown (1911); Grillner and Dubuc (1988)). In engineering literature such as Taga et al. (1991); Lewis and Bekey (2002); Marder and Bucher (2001); Yasuhiro et al. (2003), a CPG can be modeled as a nonlinear oscillator, and when placed in a feedback loop, provides a basic control architecture to achieve coordinated oscillations of engineered systems. Furthermore, in Chen et al. (2010), it was revealed how a locomotion with a natural oscillation pattern can be 


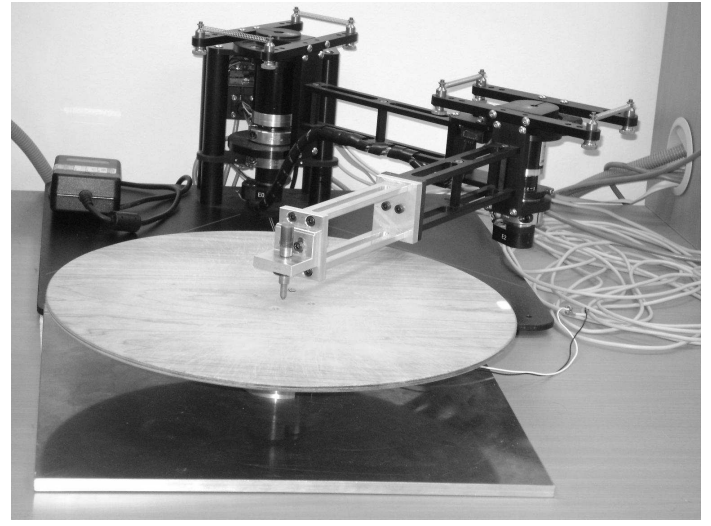

Fig. 1. The experimental PMR platform located at the University of Newcastle - a rotating disk driven by a double-link robotic arm with flexible joints.

generated autonomously. In the present paper, these ideas will be specified to the PMR model through theoretical analysis, numerical simulation and experiments. Specifically, a scenario was considered where a disk was rotated by an external force with a constant speed and two arm links oscillated as a result of the interactive force between the arm tip (finger) and the disk. In reality, however, the disk rotation speed is not applied on the system externally, but is generated by the system itself. Therefore, we will investigate how a disk rotation with a natural oscillation pattern can be generated autonomously.

The remaining sections will be organized as follows. The design and model of a PRM structure is discussed in Section 2. It is proposed in Section 3 a controller for achieving an autonomous stable disk rotation with arm links in a natural oscillation pattern. The simulated and experimental results are given in Section 4 to illustrate the effectiveness of the design.

\section{A PMR MODEL}

The PMR system shown in Fig. 1 consists of two rigid links with flexible joints and a rotating disk. Each link is driven by a DC motor through a harmonic gearbox with zero backlash. The primary link is coupled to the first drive by means of a flexible joint. It carries at its end the second harmonic drive which is coupled to the second rigid link via another flexible joint. Both motors and both flexible joints are instrumented with quadrature optical encoders. Each flexible joint stiffness can be reconfigured by using different pair of springs with different stiffness. All linear springs are from the Associated Spring Raymond. Four highresolution quadrature optical encoders from US Digital are used as sensors to measure the angular displacements of two motors and two links. The end point of second link is in touch with the disk through a finger and exerts friction force during oscillation. The disk is mounted on a base through bearing. A Faulhaber DC-tachogenerator with linear speed/back-EMF characteristics and extreme low friction is used as a sensor to measure the angular velocity of the disk. When the two links oscillate in an appropriately coordinated pattern, the under driven disk is expected to rotate with a stable speed.
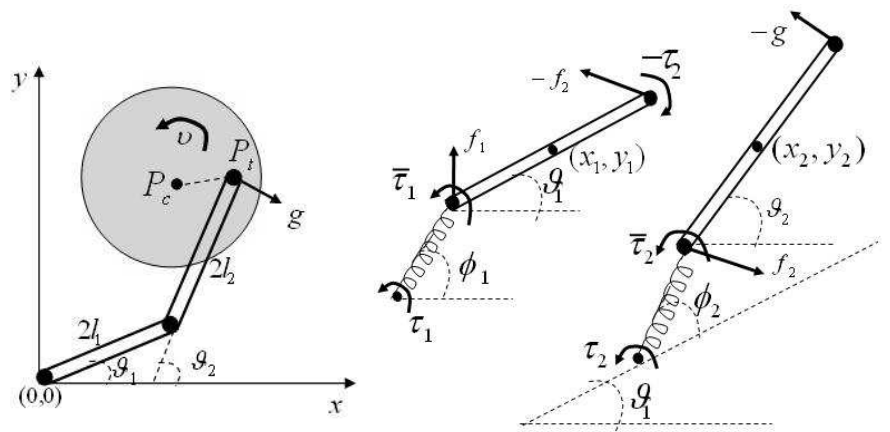

Fig. 2. Schematic diagram of the PMR system.

The schematic diagram of the PMR system is depicted in Fig. 2. We assume the link has mass $m_{i}$, length $2 l_{i}$ and moment of inertia $m_{i} l_{i}^{2} / 3$. The spring stiffness is $k_{i}$ and the motor output torque is $\tau_{i}$. The link's absolute angle is $\vartheta_{i}$ and the motor output angle is $\phi_{i}$. The subscript $i=1$ or 2 represents the quantity associated with the first or second link. For convenience, we denote vectors $m=\left[\begin{array}{ll}m_{1} & m_{2}\end{array}\right]^{\top}$ and $l, k, \tau, \vartheta, \phi$ in the same manner. Let the moment of inertia of the disk be $m_{c}$ and the rotation speed be $v$. Assume the friction force between the link tip and the disk is proportional to the relative velocity and the bearing frictional torque is proportional to the rotation speed with proportionality constants being $\mu_{t}$ and $\mu_{c}$, respectively. It is easy to see in Fig. 2 that the link tip's position is

$$
p_{t}(\vartheta)=2\left[\begin{array}{ll}
c_{\vartheta} & s_{\vartheta}
\end{array}\right]^{\top} l \text {. }
$$

When the link tip is at the disk rotation center $p_{c}$, we define the link's angle $\vartheta_{o}$ as the nominal value, i.e., $p_{c}=$ $p_{t}\left(\vartheta_{o}\right)$. And let $\theta:=\vartheta-\vartheta_{o}$. Now, it is ready to give the PMR model as follows (a similar derivation can be found in Saito et al. (2002); Blair and Iwasaki (2010), etc):

$$
\begin{aligned}
J \ddot{\theta}+D \dot{\theta}+K(v) \theta & =u \\
\dot{v}+\xi(\theta) v+\zeta(\theta, \dot{\theta}) & =0
\end{aligned}
$$

where

$$
\begin{array}{r}
J=\left(M L^{2} / 3+S_{o} F^{\top} M F S_{o}+C_{o} F^{\top} M F C_{o}\right) \\
D=4 \mu_{t} G_{o}^{\top} G_{o}, K(v)=K_{o}\left(I-E^{\top}\right)+4 \mu_{t} v G_{o}^{\top} T G_{o} \\
\xi(\theta)=\left(\mu_{c}+4 \mu_{t} \theta^{\top} P \theta\right) / m_{c}, P=G_{o}^{\top} G_{o} \\
\zeta(\theta, \dot{\theta})=-4 \mu_{t} / m_{c} \theta^{\top} Q \dot{\theta}, Q=G_{o}^{\top} T G_{o} .
\end{array}
$$

In the equations, we use the following notations:

$$
\begin{aligned}
& F=\left[\begin{array}{cc}
l_{1} & 0 \\
2 l_{1} & l_{2}
\end{array}\right], T=\left[\begin{array}{cc}
0 & 1 \\
-1 & 0
\end{array}\right], E=\left[\begin{array}{ll}
0 & 1 \\
0 & 0
\end{array}\right] \\
& K_{o}=\operatorname{diag}\{k\}, M=\operatorname{diag}\{m\}, L=\operatorname{diag}\{l\} \text {, } \\
& s_{\vartheta}=\left[\begin{array}{lll}
\sin \vartheta_{1} & \sin \vartheta_{2}
\end{array}\right]^{\top}, c_{\vartheta}=\left[\begin{array}{lll}
\cos \vartheta_{1} & \cos \vartheta_{2}
\end{array}\right]^{\top}, \\
& S_{\vartheta}=\operatorname{diag}\left\{s_{\vartheta}\right\}, C_{\vartheta}=\operatorname{diag}\left\{c_{\vartheta}\right\}, G_{\vartheta}=\left[s_{\vartheta}-c_{\vartheta}\right]^{\top} L .
\end{aligned}
$$

And we use $s_{o}=s_{\theta_{o}}, G_{o}=G_{\theta_{o}}$, etc, to keep the notation neat. In (1), we perform a preliminary control

$$
(I-E) \tau=u+K_{o}\left(I-E^{\top}\right) \vartheta_{o}-K_{o} \phi
$$

with $u$ to be designed later. The detailed model derivation is given below.

First, let $x=\left[\begin{array}{ll}x_{1} & x_{2}\end{array}\right]^{\top}$ and $y=\left[\begin{array}{ll}y_{1} & y_{2}\end{array}\right]^{\top}$ be the $x-$ and $y$-coordinates of the two link centers, that is,

$$
x=F c_{\vartheta}, y=F s_{\vartheta} \text {. }
$$


As a result,

$$
\ddot{x}=-F C_{\vartheta} \dot{\vartheta}^{2}-F S_{\vartheta} \ddot{\vartheta}, \ddot{y}=-F S_{\vartheta} \dot{\vartheta}^{2}+F C_{\vartheta} \ddot{\vartheta} .
$$

Let $f_{1}=\left[\begin{array}{ll}f_{1 x} & f_{1 y}\end{array}\right]^{\top}, f_{2}=\left[\begin{array}{ll}f_{2 x} & f_{2 y}\end{array}\right]^{\top}$ and $g=\left[\begin{array}{ll}g_{x} & g_{y}\end{array}\right]^{\top}$ be the forces on link ends, and denote $f_{x}=\left[\begin{array}{ll}f_{1 x} & f_{2 x}\end{array}\right]^{\mathrm{T}}$ and $f_{y}=\left[\begin{array}{ll}f_{1 y} & f_{2 y}\end{array}\right]^{\top}$. Then, from Newton's law for translational motion, we have, with $e:=\left[\begin{array}{ll}0 & 1\end{array}\right]^{\top}$,

$$
M \ddot{x}=(I-E) f_{x}-e g_{x}, M \ddot{y}=(I-E) f_{y}-e g_{y}
$$

or

$f_{x}=(I+E) M \ddot{x}+(I+E) e g_{x}, f_{y}=(I+E) M \ddot{y}+(I+E) e g_{y}$.

On the other hand, let $\bar{p}(\vartheta)=p_{t}(\vartheta)-p_{c}$ be the relative position between the link tip and disk center, which becomes $-T \bar{p}(\vartheta)$ with $90^{\circ}$ rotation. As a result, we have

$$
\begin{aligned}
g & =\mu_{t}\left(\dot{p}_{t}(\vartheta)+v T \bar{p}(\vartheta)\right) \\
& =2 \mu_{t}\left[-s_{\vartheta} c_{\vartheta}\right]^{\top} L \dot{\vartheta}+2 \mu_{t} v T\left[c_{\vartheta}-c_{o} s_{\vartheta}-s_{o}\right]^{\top} l
\end{aligned}
$$

Next, from Newton's law for rotational motion, we have

$$
\begin{array}{r}
M L^{2} / 3 \ddot{\vartheta}=\bar{\tau}-E \tau+L S_{\vartheta}(I+E) f_{x}-L C_{\vartheta}(I+E) f_{y} \\
+L[0 e]\left[\begin{array}{ll}
s_{\vartheta} & -c_{\vartheta}
\end{array}\right] g
\end{array}
$$

where $\bar{\tau}=\tau-K_{o}\left(\left(I-E^{\top}\right) \vartheta-\phi\right)$. Substituting the $\ddot{x}, \ddot{y}$, $f_{x}, f_{y}$ and $g$ as calculated before into this equation gives

$$
\begin{array}{r}
M L^{2} / 3 \ddot{\vartheta}=\bar{\tau}-E \tau+S_{\vartheta} F^{\top} M \ddot{x}-C_{\vartheta} F^{\top} M \ddot{y}+S_{\vartheta} F^{\top} e g_{x} \\
-C_{\vartheta} F^{\top} e g_{y}+L\left[\begin{array}{ll}
0 & e
\end{array}\right]\left[\begin{array}{ll}
s_{\vartheta} & -c_{\vartheta}
\end{array}\right] g,
\end{array}
$$

where we note $L(I+E)^{2}=F^{\top}$. Then,

$M L^{2} / 3 \ddot{\vartheta}=\bar{\tau}-E \tau+S_{\vartheta} F^{\top} M \ddot{x}-C_{\vartheta} F^{\top} M \ddot{y}+2 L\left[s_{\vartheta}-c_{\vartheta}\right] g$ and

$$
\begin{array}{r}
M L^{2} / 3 \ddot{\vartheta}=(I-E) \tau-K_{o}\left(\left(I-E^{\top}\right) \vartheta-\phi\right)+ \\
S_{\vartheta} F^{\top} M\left[-F C_{\vartheta} \dot{\vartheta}^{2}-F S_{\vartheta} \ddot{\vartheta}\right]-C_{\vartheta} F^{\top} M\left[-F S_{\vartheta} \dot{\vartheta}^{2}+F C_{\vartheta} \ddot{\vartheta}\right] \\
+2 L\left[s_{\vartheta}-c_{\vartheta}\right]\left[2 \mu_{t}\left[-s_{\vartheta}, c_{\vartheta}\right]^{\top} L \dot{\vartheta}\right. \\
\left.+2 \mu_{t} v T\left[c_{\vartheta}-c_{o} s_{\vartheta}-s_{o}\right]^{\top} l\right] .
\end{array}
$$

The linearized system at $\vartheta=\vartheta_{o}$ is

$$
\begin{array}{r}
M L^{2} / 3 \ddot{\theta}=(I-E) \tau-K_{o}\left(I-E^{\top}\right) \theta-K_{o}\left(I-E^{\top}\right) \vartheta_{o} \\
+K_{o} \phi-S_{o} F^{\top} M F S_{o} \ddot{\theta}-C_{o} F^{\top} M F C_{o} \ddot{\theta}-4 \mu_{t} G_{o}^{\top} G_{o} \dot{\theta} \\
-4 \mu_{t} v G_{o}^{\top} T G_{o} \theta
\end{array}
$$

where we note $\left[c_{\vartheta}-c_{o} s_{\vartheta}-s_{o}\right]^{\top} l \approx-G_{o} \theta$.

The dynamics of the disk is governed by Newton's law for rotational motion is given as follows:

$$
\begin{aligned}
& m_{c} \dot{v}=-\mu_{c} v-[T \bar{p}(\vartheta)]^{\top} g=-\mu_{c} v+\bar{p}^{\top}(\vartheta) T g \\
& =-\mu_{c} v+2 l^{\top}\left[\begin{array}{ll}
c_{\vartheta}-c_{o} & s_{\vartheta}-s_{o}
\end{array}\right] \\
& T\left[2 \mu_{t}\left[-s_{\vartheta} c_{\vartheta}\right]^{\top} L \dot{\vartheta}+2 \mu_{t} v T\left[c_{\vartheta}-c_{o} s_{\vartheta}-s_{o}\right]^{\top} l\right] .
\end{aligned}
$$

Then, the linearized model is

$$
m_{c} \dot{v}=-\mu_{c} v+4 \mu_{t} \theta^{\top} G_{o}^{\top} T G_{o} \dot{\theta}-4 \mu_{t} v \theta^{\top} G_{o}^{\top} G_{o} \theta .
$$

So the torque input $\tau$ generates periodic motion of two links that interacts with the disk to yield its rotational velocity $v$. thus the PMR represents typical dynamics of animal locomotion.

\section{CONTROLLER DESIGN}

As developed in the previous section, the locomotion system under investigation is composed of the two equations (1) and (2). The major objective is to find an effective controller $u$ such that the closed-loop system can achieve two properties: (i) the double-link $(\theta)$ displays a natural oscillation; and (ii) a desired rotation velocity $v$ is generated by the undulatory motion of the links in natural oscillation. Corresponding to the two properties, the controller design and analysis are given below.

\subsection{Natural Oscillation of Links}

We will first consider the system (1), and define its natural oscillation in terms of the state $\theta$. Let us revisit the definition of natural oscillation given in Chen and Iwasaki (2009).

Definition 1. Consider the system (1) with the damping effect adjusted by a parameter $\epsilon \in \mathbb{R}$ and define the modified system with no input:

$$
J \ddot{\theta}-\epsilon J \dot{\theta}+K \theta=0 .
$$

If this system has a nonzero characteristic root on the imaginary axis $\lambda= \pm j \omega$ with associated mode shape $z$ for a specific value $\epsilon:=\rho$, then the corresponding natural motion of $(3)$ is called a natural oscillation $(\omega, z)$ of the original system (1) with damping factor $\rho$, where $\omega$ and $z$ are referred to as the natural frequency and mode shape of the natural oscillation.

The natural oscillation can be explicitly calculated using the following lemma given in Chen and Iwasaki (2009).

Lemma 1. Consider the system in (1). Let $\omega, \rho \in \mathbb{R}$ and $z \in \mathbb{C}^{2}$ be given. Then, $(\omega, z)$ is a natural oscillation of (1) with damping factor $\rho$ if and only if $(\omega, z, \rho) \in \mathbb{N}$ where

$$
\begin{aligned}
\mathbb{N}:=\{(\omega, z, \rho) & \in \mathbb{R} \times \mathbb{C}^{2} \times \mathbb{R}: \omega=\sqrt{\Re(\varsigma)}, \\
\rho & \left.=\mu+\frac{\Im(\varsigma)}{\sqrt{\Re(\varsigma)}},(\varsigma, z) \in \mathbb{M}\right\}
\end{aligned}
$$

and $\mathbb{M}$ is the set of eigenvalue/eigenvector pairs of $M:=$ $J^{-1} K$ :

$$
\mathbb{M}:=\left\{(\varsigma, z) \in \mathbb{C} \times \mathbb{C}^{2}:(\varsigma-M) z=0\right\} .
$$

Let $\varrho$ be the smallest damping factor to which there corresponds a natural oscillation:

$$
\varrho:=\min _{(\varsigma, z) \in \mathbb{M}} \mu+\frac{\Im(\varsigma)}{\sqrt{\Re(\varsigma)}} .
$$

Suppose the minimizer is unique. We focus on the natural oscillation with the smallest damping factor $\varrho$, and will refer to it simply as the natural oscillation. The main task is to design a feedback controller for the following natural entrainment problem.

Natural Entrainment Problem: Consider the system (1). Let the orbit of the natural oscillation $(\omega, z)$ be defined by

$$
\begin{array}{r}
\mathbb{O}:=\left\{(\vartheta(t), \dot{\vartheta}(t)) \in \mathbb{R}^{2} \times \mathbb{R}^{2} \mid t \in \mathbb{R}\right\}, \\
\vartheta(t):=Z \sin (\omega t+\gamma) .
\end{array}
$$


${ }^{1}$ A controller is said to achieve exact entrainment to the natural oscillation $(\omega, z)$ if the following property holds: When the initial condition $(\theta(0), \dot{\theta}(0))$ is sufficiently close to the orbit $\mathbb{O}$, i.e., when $\min _{\left(\vartheta_{o}, \dot{\vartheta}_{o}\right) \in \mathbb{O}}\left\|\theta(0)-\vartheta_{o}\right\|+\| \dot{\theta}(0)-$ $\dot{\vartheta}_{o} \|$ is sufficiently small, the trajectory of the closedloop system $\phi(t)$ converges to the orbit $\mathbb{O}$, i.e., there exists $t_{o}$, dependent upon the initial condition, such that $\lim _{t \rightarrow \infty} \theta(t)-\vartheta\left(t+t_{o}\right)=0$.

Inspired by the CPG control mechanism in animal locomotion, some nonlinear controllers have been constructed for the problem of entrainment to natural oscillation. For example, a slightly modified positive derivative feedback controller is borrowed from Chen and Iwasaki (2009) as follows.

Theorem 2. Consider the system (1). Let $(\omega, z)$ be the natural oscillation with damping factor $\varrho$ as described in Lemma 1. Let $\epsilon, \eta \in \mathbb{R}$ be such that $\eta>0, \epsilon<\varrho$. Then a positive parameter

$$
r:(\varrho-\epsilon) /(\kappa(\eta \omega) \eta)
$$

satisfies $r>\omega(\varrho-\epsilon)$ where $\kappa$ is the describing function of $\psi$. And the controller

$$
u=D \dot{\theta}+\epsilon J \dot{\theta}+r J Z \psi\left(\eta Z^{-1} \dot{\theta}\right) .
$$

is expected to achieve the entrainment to the natural oscillation $(\omega, z)$.

Remark 1. In (5), the term $w=r J Z \psi\left(\eta Z^{-1} \dot{\theta}\right)$ takes the following form:

$$
w=G \psi(q), \quad q=f(s) H \theta
$$

where $G$ and $H$ are $2 \times 2$ real matrices, $f(s)$ is a scalar transfer function (in particular, $f(s)=s$ for the positive derivative feedback controller), and $\psi$ is a static nonlinearity satisfying the following properties:

- $\psi$ is odd, bounded, and strictly increasing.

- $\psi(x)$ is strictly concave on $x>0$, and $\psi^{\prime}(0)=1$.

The structure in (6) is motivated by biological CPG control mechanisms. In particular, the simplest inputoutput model of neuronal dynamics is given by $v_{\text {post }}=$ $\psi\left(f(s) v_{\text {pre }}\right)$ from the presynaptic potential $v_{\text {pre }}$ to the postsynaptic potential $v_{\text {post }}$ where $\psi$ and $f(s)$ represent the threshold nonlinearity and dynamics (time lag, adaptation, etc.) associated with synaptic and cell membrane processes, respectively. The controller in (6) is a network of multiple neurons with the interconnections specified by $G$ and $H$.

\subsection{Rotation of Disk}

Now, the double link pendulum in natural oscillation has been achieved by an entrainment controller inspired by a CPG based structure. In the formulation of natural oscillation and the control design procedure, the rotation speed $v$ is assumed to be externally imposed. Actually, a more interesting question of the locomotion system is that the introduced natural oscillation in turn generates the velocity $v$. Next, we will analyze how the rotation speed

1 Throughout the paper, we use the notation $Z e^{j \gamma}:=z$ to define $Z$ to be the diagonal matrix with $\left|z_{i}\right|$ on the $i^{\text {th }}$ diagonal entry, $\gamma$ to be the vector with $\angle z_{i}$ in the $i^{\text {th }}$ entry, and $e^{j \gamma}$ to be the vector with $e^{j \gamma_{i}}$ stacked in a column. $v$ is induced by the link oscillation. An assumption is first imposed to facilitate the analysis:

Assumption 1. For the CPG controller based on a given velocity $v_{0}$, the closed-loop system is sufficiently fast to achieve the natural oscillation associated with any $v$ in the neighborhood of $v_{0}$.

For a natural oscillation $(\omega, z)$, depending on the velocity $v$, we can assume $\theta(t)=\Re\left(z e^{j \omega t}\right)$, and hence,

$$
\dot{\theta}(t)=\Re\left(j \omega z e^{j \omega t}\right)+\delta(\dot{z}, \dot{\omega})
$$

for some terms $\delta_{1}$ and $\delta_{2}$ vanishing at $(\dot{z}, \dot{\omega}, \ddot{z}, \ddot{\omega})=0$. Then, some direct calculation is given below:

$$
\begin{aligned}
& \theta^{\top} P \theta=|z|^{\top} \bar{P}|z|+|z|^{\top} \tilde{P}(t)|z| \\
& \theta^{\top} Q \dot{\theta}=-\omega|z|^{\top} \bar{Q}|z|-\omega|z|^{\top} \tilde{Q}(t)|z|+\theta^{\top} Q \delta
\end{aligned}
$$

and

$$
\begin{aligned}
\bar{P}_{i j} & =P_{i j} \cos \left(\phi_{i}-\phi_{j}\right) / 2 \\
\tilde{P}_{i j}(t) & =P_{i j} \cos \left(\phi_{i}+\phi_{j}+2 \omega t\right) / 2, \\
\bar{Q}_{i j} & =-Q_{i j} \sin \left(\phi_{i}-\phi_{j}\right) / 2, \\
\tilde{Q}_{i j}(t) & =Q_{i j} \sin \left(\phi_{i}+\phi_{j}+2 \omega t\right) / 2
\end{aligned}
$$

where $|z|$ is the column vector with entries $\left|z_{i}\right|$. By using this calculation, we can rewrite (2) as

$$
\dot{v} /\|z\|^{2}+[\bar{\xi}(v)+\tilde{\xi}(v, t)] v+\bar{\zeta}(v)+\tilde{\zeta}(v, t)=0
$$

where $c_{c}=\mu_{c} / m_{c}$ and $c_{t}=\mu_{t} / m_{c}$, and

$$
\begin{aligned}
\bar{\xi}(v) & =c_{c} /|| z \|^{2}+4 c_{t}\left|z_{o}\right|^{\top} \bar{P}\left|z_{o}\right| \\
\tilde{\xi}(v, t) & =4 c_{t}\left|z_{o}\right|^{\top} \tilde{P}(t)\left|z_{o}\right| \\
\bar{\zeta}(v) & =4 c_{t} \omega\left|z_{o}\right|^{\top} \bar{Q}\left|z_{o}\right| \\
\tilde{\zeta}(v, t) & =4 c_{t} \omega\left|z_{o}\right|^{\top} \tilde{Q}(t)\left|z_{o}\right|-4 c_{t} \theta^{\top} Q \delta .
\end{aligned}
$$

Here, we use $z_{o}:=z /\|z\|$ with $\left\|z_{o}\right\|=1$ which is the normalized eigenvector representing the oscillation phases and relative amplitudes. The quantities, in particular, $\omega, z_{o}$, and $\bar{Q}$ in the above development, depend on the velocity $v$, and will be explicitly expressed as $\omega(v), z_{o}(v)$, and $\bar{Q}(v)$ when the dependence is important.

Clearly, for a given $v, \bar{\xi}(v)$ and $\bar{\zeta}(v)$ are two constants, but $\tilde{\xi}(v, t)$ and $\tilde{\zeta}(v, t)$ are sinusoidally time varying functions of frequency $2 \omega$. With the decomposition of the functions $\xi$ and $\zeta$, we can decompose the velocity $v=\bar{v}+\tilde{v}$ in a corresponding manner. Let $\bar{v}$ be governed by the dynamics:

$$
\dot{\bar{v}} /\|z\|^{2}+\bar{\xi}(\bar{v}) \bar{v}+\bar{\zeta}(\bar{v})=0,
$$

then $\tilde{v}$ must be governed by

$$
\begin{aligned}
& \dot{\tilde{v}} /\|z\|^{2}=-\bar{\xi}(v) \tilde{v}+[\bar{\xi}(\bar{v})-\bar{\xi}(v)] \bar{v} \\
& +[\bar{\zeta}(\bar{v})-\bar{\zeta}(v)]-\tilde{\xi}(v, t) v-\tilde{\zeta}(v, t) .
\end{aligned}
$$

We call $\bar{v}$ and $\tilde{v}$ the average velocity and ripple, respectively. It has been shown in Chen et al. (2010) that the magnitude of the ripple $\tilde{v}$ is expected to be small if the oscillation amplitude is small. Now, it is ready to reach the following conclusion on the average velocity $\bar{v}$ in the following theorem. 
Theorem 3. Under Assumption 1, the average velocity $\bar{v}$ governed by (8) locally asymptotically converges to $\bar{v}=v_{o}$ if $\alpha\left(v_{o}\right)=v_{o}$ and $\alpha^{\prime}\left(v_{o}\right)<1$, where

$$
\alpha(x):=\frac{-4 c_{t} \omega\left|z_{o}\right|^{\top} \bar{Q}\left|z_{o}\right|}{c_{c} /\|z\|^{2}+4 c_{t}\left|z_{o}\right|^{\top} \bar{P}\left|z_{o}\right|} .
$$

\section{SIMULATION AND EXPERIMENT}

Consider the PMR system with the following parameter values. The two links have masses of $m 1=2.42 \mathrm{~kg}$ and $m_{2}=1.95 \mathrm{~kg}$, and lengths of $2 l_{1}=0.35 \mathrm{~m}$ and $2 l_{2}=0.45 \mathrm{~m}$. The disk rotational inertia is $m_{c}=2 \mathrm{kgm}^{2}$. The nominal link angles are $\vartheta_{o}=\left[25^{\circ},-25^{\circ}\right]$. The environmental force constants are $\mu_{t}=18 \mathrm{~kg} / \mathrm{s}$ and $\mu_{c}=0 \mathrm{Ns} / \mathrm{m}$. We consider two sets of joint stiffness $k=[3.8,3]^{\top} \mathrm{kgm}^{2} / \mathrm{s}^{2} \mathrm{rad}$ and $k=[6.8,4]^{\top} \mathrm{kgm}^{2} / \mathrm{s}^{2} \mathrm{rad}$ to examine the effect of stiffness perturbation. For different $k$ 's, we can find the solution to $\alpha(x)=x$ in Fig. 3. The result shows that, to expect a higher velocity, the stiffness of the body must be strengthen. From Fig. 3, it is easy to see that $\alpha^{\prime}\left(v_{o}\right)<1$ is true for both cases. In the other words, both equilibrium points $v_{o}=4.6 \mathrm{rad} / \mathrm{s}$ and $v_{o}=5.8 \mathrm{rad} / \mathrm{s}$ corresponding to $k=[3.8,3]^{\top}$ and $k=[6.8,4]^{\top}$ are asymptotically stable. Moreover, the natural oscillation patterns for these two cases are listed in Table 1 . Now we can see in Fig. 4 and Fig. 6 that the controller very closely achieves theoretical velocity in numerical simulation. Moreover, the experimental results are shown in Fig. 5 and Fig. 7. All the theoretical, simulated and experimental results are cross checked with respect to theoretical values as shown in Table 1 in terms of disk speed, link oscillation amplitudes, period, and phase difference. It is seen that all results shown in Table 1 are reasonably consistent.

Table 1. Theoretical/simulated/experimental locomotion profiles with different stiffness

\begin{tabular}{|l||c|c|c|c|c|}
\hline$k=[3.8,3]^{\top}$ & $v(\mathrm{rad} / \mathrm{sec})$ & $\left|z_{1}\right|$ & $\left|z_{2}\right|$ & Period(s) & Phase \\
\hline Theo. & 4.6 & $9.0^{\circ}$ & $10.3^{\circ}$ & 1.21 & $130^{\circ}$ \\
Simu. & 4.5 & $9.3^{\circ}$ & $10.6^{\circ}$ & 1.25 & $130^{\circ}$ \\
Exp. & 5.0 & $7.9^{\circ}$ & $10.0^{\circ}$ & 1.21 & $136^{\circ}$ \\
\hline \hline$k=[6.8,4]^{\top}$ & $v(\mathrm{rad} / \mathrm{sec})$ & $\left|z_{1}\right|$ & $\left|z_{2}\right|$ & Period(s) & Phase \\
\hline Theo. & 5.8 & $9.3^{\circ}$ & $10.1^{\circ}$ & 1.00 & $129^{\circ}$ \\
Simu. & 5.7 & $9.2^{\circ}$ & $9.9^{\circ}$ & 1.02 & $129^{\circ}$ \\
Exp. & 6.0 & $8.7^{\circ}$ & $11.4^{\circ}$ & 1.01 & $136^{\circ}$ \\
\hline
\end{tabular}

\section{CONCLUSION}

In this paper, we discussed the design of a PMR structure and a CPG based controller for the generation of stable disk rotation with natural oscillation of links. The phenomena is based on the simple PRM structure but it is expected to capture the fundamental principle underlying the locomotion behaviors of natural systems or complicated engineered systems. The CPG based controller is examined by the cross check among theoretical analysis, numerical simulation, and experiments. The approach is expected to extend to more complicated systems like multi-link snake-like robots.

\section{REFERENCES}

Blair, J. and Iwasaki, T. (2010). Optimal gaits for mechanical rectifier systems. IEEE Trans. Auto. Contr., to appear.
Brown, T. (1911). The intrinsic factors in the act of progression in the mammal. Proceedings of the Royal Society of London. Series B, Containing Papers of a Biological Character, 84(572), 308-319.

Cavagna, G., Heglund, N., and C.Taylor. (1977). Mechanical work in terrestrial locomotion: Two basic mechanisms for minimizing energy expenditure. Am. J. Physiol., 233, R243-R 261.

Chen, Z. and Iwasaki, T. (2009). Robust entrainment to natural oscillations of asymmetric systems arising from animal locomotion. Proceedings of the 48th IEEE Conference on Decision and Control, $2954-2959$.

Chen, Z., Zhu, L., and Iwasaki, T. (2010). Autonomous locomotion of multi-link mechanical systems via natural oscillation pattern. Proceedings of the 49th IEEE Conference on Decision and Control.

Futakata, Y. and Iwasaki, T. (2008). Formal analysis of resonance entrainment by central pattern generator. Journal of Mathematical Biology, 57, 183-207.

Grillner, S. and Dubuc, R. (1988). Control of locomotion in vertebrates: spinal and supraspinal mechanisms. Advances in Neurology, 47, 425-453.

Hatsopoulos, N. (1996). Coupling the neural and physical dynamics in rhythmic movements. Neural Computation, $8(3), 567-581$.

Iwasaki, T. and Liu, B. (2004). Feedback control with central pattern generator for decentralized coordination of prototype mechanical rectifier. Proceeding of American Control Conference, 11(23), 3059-3064.

Iwasaki, T. and Zheng, M. (2006). Sensory feedback mechanism underlying entrainment of central pattern generator to mechanical resonance. Biological Cybernetics, 94, 245-261.

Lewis, M. and Bekey, G. (2002). Gait adaptation in a quadruped robot. Autonomous Robots, 12, $301-312$.

Marder, E. and Bucher, D. (2001). Central pattern generators and the control of rhythmic movements. Current Biology, 11(23), 986-996.

Saito, M., Fukaya, M., and Iwasaki, T. (2002). Serpentine locomotion with robotic snakes. IEEE Control Systems Magazine, 22(1), 64-81.

Taga, G., Yamaguchi, Y., and Shimizu, H. (1991). Selforganized control of bipedal locomotion by neural oscillators in unpredictable environment. Biological Cybernetics, 65, 147-159.

Verdaasdonk, B., Koopman., H., and Helm, F. (2007). Resonance tuning in a neuro-musculo-skeletal model of the forearm. Biological Cybernetics, 96, 165-180.

Williamson, M. (1998). Neural control of rhythmic arm movements. Neural Networks, 11(7-8), 1379 - 1394.

Yasuhiro, F., Hiroshi, K., and Cohen., A. (2003). Adaptive dynamic walking of a quadruped robot on irregular terrain based on biological concepts. International Journal of Robotics Research, 22(3-4), 187 - 202. 


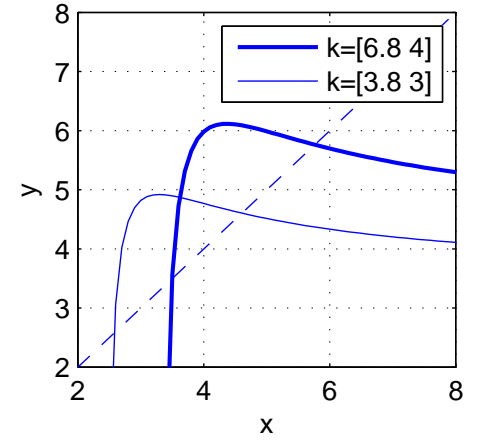

Fig. 3. Solution to $\alpha(x)=x$. The solid curves represent $y=\alpha(x)$ for $k=[3.8,3]^{\top}$ and $k=[6.8,4]^{\top}$, and the dashed line is $y=x$. The intersection $\alpha(x)=x$ occurs at $x=4.5$ and $x=5.8$, respectively.
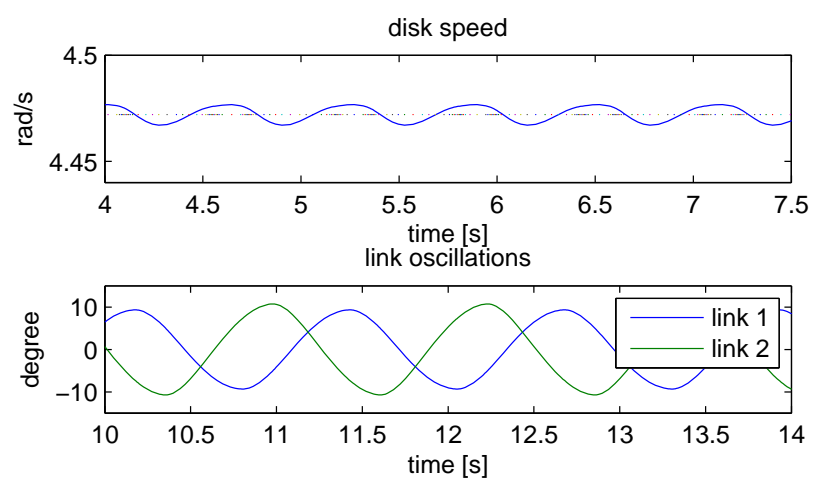

Fig. 4. Simulation profile with $k=[3.8,3]^{\top}$. Above: selfgenerated disk velocity $v$. Below: undulatory trajectories $\theta_{1}$ and $\theta_{2}$ of two links.

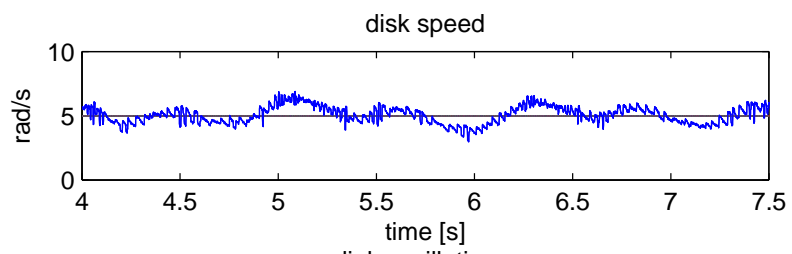

link oscillations

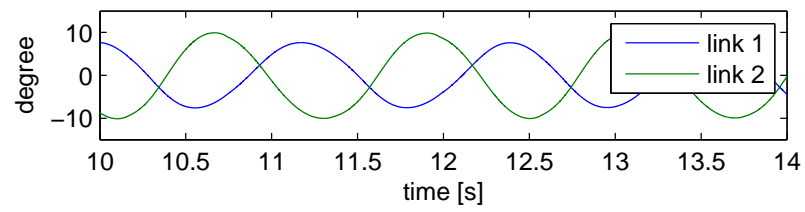

Fig. 5. Experimental profile with $k=[3.8,3]^{\top}$. Above: selfgenerated disk velocity $v$. Below: undulatory trajectories $\theta_{1}$ and $\theta_{2}$ of two links.

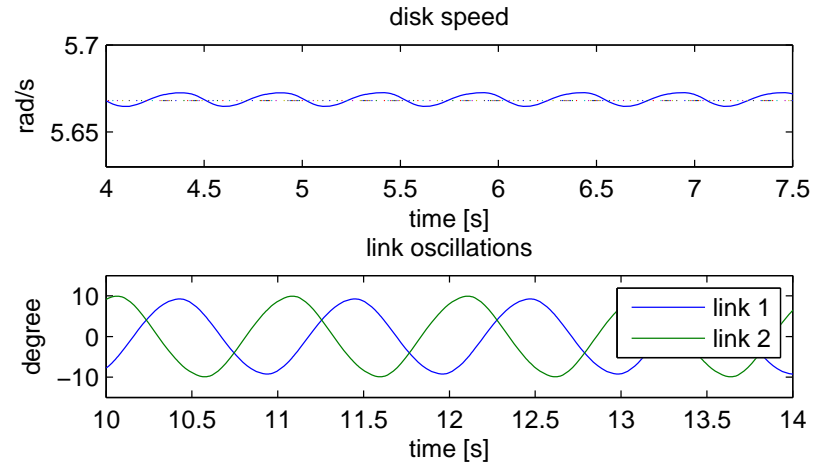

Fig. 6. Simulation profile with $k=[6.8,4]^{\top}$. Above: selfgenerated disk velocity $v$. Below: undulatory trajectories $\theta_{1}$ and $\theta_{2}$ of two links.
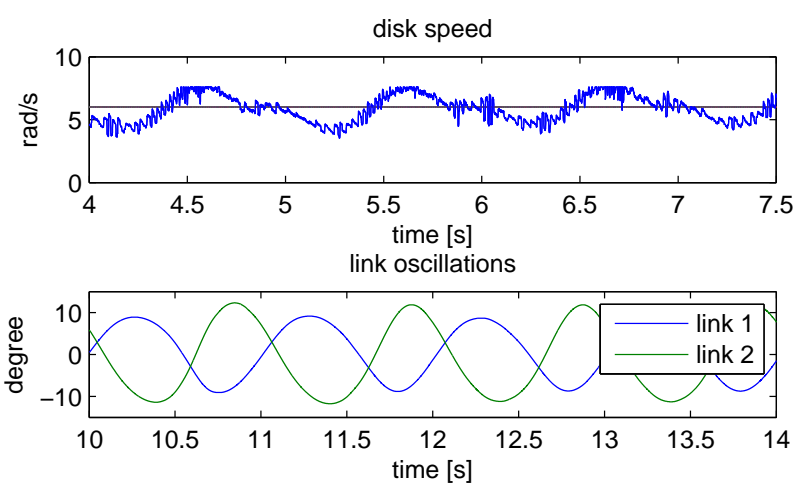

Fig. 7. Experimental profile with $k=[6.8,4]^{\top}$. Above: selfgenerated disk velocity $v$. Below: undulatory trajectories $\theta_{1}$ and $\theta_{2}$ of two links. 